\title{
Internet Connection Control based on Idle Time Using User Behavior Pattern Analysis
}

\section{Fadilah Fahrul Hardiansyah, Joko Lianto Buliali, Waskitho Wibisono}

\author{
Faculty of Informatics Engineering \\ Institut Teknologi Sepuluh Nopember \\ Email: fahrul@pens.ac.id, joko@cs.its.ac.id,waswib@gmail.com
}

\begin{abstract}
The increase of smartphone ability is rapidly increasing the power consumption. Many methods have been proposed to reduce smartphone power consumption. Most of these methods use the internet connection control based on the availability of the battery power level regardless of when and where a waste of energy occurs. This paper proposes a new approach to control the internet connection based on idle time using user behavior pattern analysis. User behavior patterns are used to predict idle time duration. Internet connection control performed during idle time. During idle time internet connection periodically switched on and off by a certain time interval. This method effectively reduces a waste of energy. Control of the internet connection does not interfere the user because it is implemented on idle time.
\end{abstract}

Keywords: Smartphone, User Behavior, Pattern Recognition, Idle Time, Internet Connection Control

\section{INTRODUCTION}

Many efforts have been attempted to overcome the high energy usage for smartphone. This efforts is done in various aspects including devices and software. But this effort becomes useless if the usage of energy is not appropriate. The usage of internet relentlessly, background process that always running, SNS (Social Network Service) that always synchronize to the server consume a lot of energy. Energy consumption for these processes continue even though the device is in idle condition [1].

Many approaches and methods have been proposed. Including a simple method such as manual setting and configuration from users [2] and a lot of complex methods such as Smart offloading methods [3], selective data reception methods [4], and signaling interval control [5].

Most of these methods use the internet connection control based on the availability of the battery power level regardless of when and where a waste of energy occurs. Because these methods only based on the availability of the battery energy, when the battery level is high energy saving process is low. If 
the battery level is low many services and process is terminated which can interfere user's convenience, whereas low battery level does not mean the user does not need the smartphone. Energy saving should be done when the user is not using a smartphone or smartphone devices in idle condition. Idle condition is the most common condition in smartphones [6]. Energy is still consumed at idle time [1] while the user is not using the device.

This paper proposes a new approach to control the internet connection based on idle time using user behavior pattern analysis. Control of the internet connection worked during idle time. We define idle time based on user activity observed through CPU usage levels. The duration of idle time is predicted by recognizing user behavior patterns. During idle time internet connection periodically switched on and off by a certain time interval.

\section{RELATED WORKS}

Many methods have been attempted to overcome the high energy usage of smartphone, one of these methods is Smart Offloading [3]. This method predicts energy consumption and execution time for data transmission process. Offloading is all steps in data transfer process. Offloading started from the request until receiving the data. This method determines whether the offloading process is executed or not based on estimates of energy consumption and execution time.

Selective data reception is another method proposed by Min Woo Kim [4]. When user's device receives a packet, the system will check the size of that packet. If the packet size is less than the threshold then it will run in normal mode, but if it exceeds the threshold the system will check the battery capacity. Packet transmission canceled if the battery capacity is weak. This method is very effective to extend the battery life time when the battery energy level are critical.

There are other approaches to control the interval of sending and receiving data packets when the battery capacity is insufficient [5]. This method is very effective to save battery energy, especially when the battery level is low. The lower battery capacity, the longer interval, and greater the energy saved. When entering power saving mode smartphone can still perform data transfer process periodically according to the given interval. But the interval can be very long.

Our proposed method uses intervalling idea of that method. The interval is given on an internet connection at idle time. Periodically internet connection is turn on and off based on the given interval. The interval duration depends on the idle Time duration. To predict the idle duration we analyze the user behavior.

\section{ORIGINALITY}

This paper proposes a new approach to control the internet connection based on idle time using user behavior pattern analysis. The internet connection control is performed during idle time. We define idle time based on user activity observed through CPU usage levels. The duration of idle time 
is predicted by recognizing user behavior patterns. During the idle time internet connection periodically switched on and off by a certain time interval.

\section{SYSTEM DESIGN}

A new approach is proposed to reduce energy consumption on a smartphone. Energy consumption is reduced by controlling the Internet connection on idle time. We specify the idle time based on the level of CPU usage. Low CPU level means smartphone on idle condition. Idle time may occur with highly varied duration. To determine idle duration we use the user patterns analysis. Idle duration is used to determine how long the battery saving mode occurs and how long interval of the internet connection.

There are four main processes in this method: collecting of data learning, feature extraction, learning process and matching process. Collecting of data conducted by monitoring the input parameters (time, CPU usage, upload, download, and coordinates). The feature extraction is performed on the collected data. Learning process is using the result of data extraction. Data are clustered into several groups based on the idle time. In the matching process, system monitors the smartphone activity while active. When smartphone on idle time, activity data that collected previously is extracted to get the feature. Feature data is used to predict how long idle time occurs.

\subsection{Collecting Data}

On this process, system records user activity on smartphone. The recorded data consisting of time, CPU_usage, upload, download, latitude, and longitude. These data are used because it has high correlation to the user activity. We can determine idle and active condition based on CPU_usage, upload, and download. When the smartphone is used, smartphone CPU usage is definitely high. But when there is on activity on smartphone, CPU usage is low. Latitude and longitude are coordinate location (latitude and longitude) of device when data is recorded. Smartphone coordinate taken from GPS sensor on smartphone. Latitude value range is -90 to 90 , while the longitude value range is -180 to 180 . Latitude and longitude also have a correlation to the user's activity. User location related to user activity [7], and of course also affect the user's habits.

This process is performed by services running behind the scenes. Smartphone monitoring process records the data every 15 seconds. In one day data collected about 5760 data. This monitoring process is done in a month to get good data for training. The data collected in this process is about 40 thousand data. Table 1 is an example of the recorded data.

Time on Table 1 is time when data collected. Units used for time data is minutes. In one day there is 1440 . Here is the time calculation formula.

$\boldsymbol{t}=\boldsymbol{h} * \mathbf{6 0}+\boldsymbol{m}$

Where: $t$ is time, $h$ is hour, and $m$ is minute. 
Table 1. Smartphone Activity Data Monitoring

\begin{tabular}{|c|c|c|c|c|c|}
\hline Time & CPU_usage & Upload & Download & Latitude & Longitude \\
\hline 1412 & 93 & 0 & 0 & -7.2453575 & 112.759956 \\
\hline 1412 & 87 & 0 & 0 & -7.2453594 & 112.759955 \\
\hline 1412 & 62 & 0 & 0 & -7.2453599 & 112.759955 \\
\hline 1412 & 34 & 0 & 0 & -7.2453599 & 112.759955 \\
\hline 1413 & 39 & 0 & 0 & -7.2453591 & 112.759956 \\
\hline 1413 & 32 & 0 & 0 & -7.2453591 & 112.759952 \\
\hline 1413 & 39 & 0 & 0 & -7.2453586 & 112.759951 \\
\hline 1413 & 18 & 0 & 0 & -7.2453586 & 112.759951 \\
\hline 1414 & 100 & 0 & 0 & -7.2453594 & 112.759952 \\
\hline 1414 & 39 & 0 & 0 & -7.2453594 & 112.759952 \\
\hline
\end{tabular}

CPU_usage is percentage of CPU usage in the period of data monitoring. Download and upload is data traffic in the period of data monitoring. Latitude and longitude are coordinate location (latitude and longitude) of device when data is recorded. Smartphone coordinate is taken from GPS sensor on smartphone.

\subsection{Feature Extraction}

This process is conducted to get features from collected data. It start with checking idle status from every data. Idle and active data shown on CPU_usage. When CPU_usage over the threshold means data is active. Threshold used on CPU_usage is $25 \%$. Table 2 is an example of data that the idle status has been checked.

Table 2. Smartphone Activity Data Monitoring

\begin{tabular}{|c|c|c|c|c|c|c|}
\hline Time & CPU_usage & Up & Down & Latitude & Longitude & Idle \\
\hline 812 & 75 & 0 & 0 & -7.2453575 & 112.759956 & 1 \\
\hline 812 & 87 & 0 & 0 & -7.2453594 & 112.759955 & 1 \\
\hline 812 & 62 & 0 & 0 & -7.2453599 & 112.759955 & 1 \\
\hline 812 & 34 & 0 & 0 & -7.2453599 & 112.759955 & 1 \\
\hline 813 & 30 & 0 & 0 & -7.2453591 & 112.759956 & 1 \\
\hline 813 & 28 & 0 & 0 & -7.2453591 & 112.759952 & 1 \\
\hline 813 & 17 & 0 & 0 & -7.2453586 & 112.759951 & 0 \\
\hline 813 & 5 & 0 & 0 & -7.2453586 & 112.759951 & 0 \\
\hline 814 & 0 & 0 & 0 & -7.2453594 & 112.759952 & 0 \\
\hline 814 & 0 & 0 & 0 & -7.2453594 & 112.759952 & 0 \\
\hline
\end{tabular}

Table 2 is an example of data that the idle status has been checked. Value 1 on idle column means active data, while 0 means idle. A data pattern 
get from an active period and idle period. Figure 1 is data illustration for a pattern.

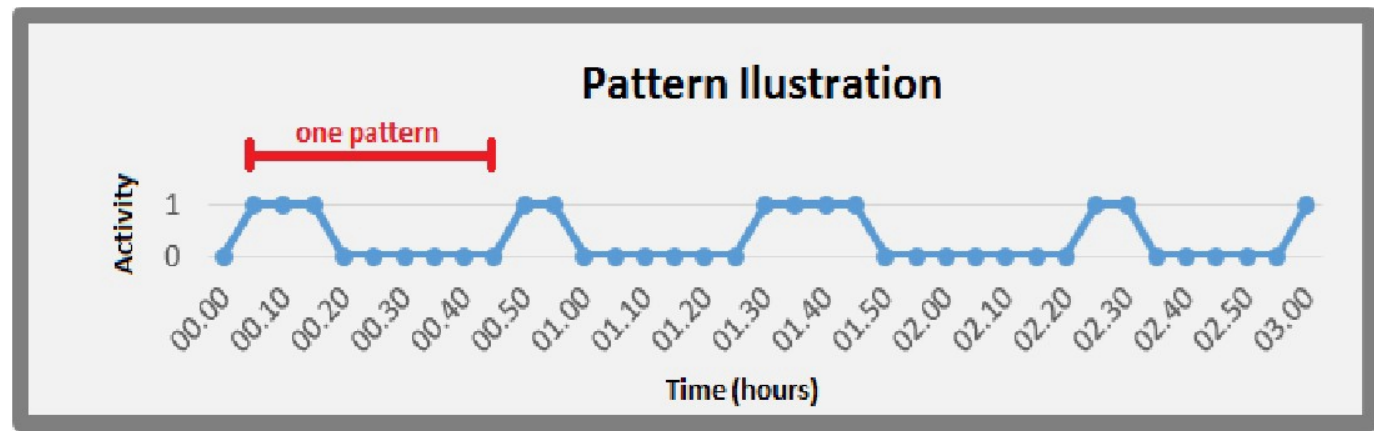

Figure 1. Pattern Illustration

Every point on Figure 1 represent a data. Every point have value 1 (active) or 0 (idle). A data pattern is group of data in an active period and idle period. Every pattern have time attribute, CPU_usage, download, upload, longitude, latitude, idle duration. Value of the attribute collected by extracting feature of an active data period using average method. Table 3 is data pattern after extraction. Idle duration represent the length of idle time that occurs in a pattern. Idle duration obtained by calculating idle period on the pattern.

Table 3. Data Pattern

\begin{tabular}{|c|c|c|c|c|c|c|}
\hline Time & CPU & Up & Down & Latitude & Longitude & Idle_duration \\
\hline 1405 & 50 & 3 & 1 & -7.2453575 & 112.759956 & 0 \\
\hline 1407 & 74 & 19 & 8 & -7.2453594 & 112.759955 & 0 \\
\hline 1408 & 29 & 1 & 1 & -7.2453599 & 112.759955 & 0 \\
\hline 1413 & 69 & 1 & 0 & -7.2453599 & 112.759955 & 1 \\
\hline 1417 & 42 & 0 & 0 & -7.2453591 & 112.759956 & 0 \\
\hline 1426 & 37 & 0 & 0 & -7.2453591 & 112.759952 & 284 \\
\hline 270 & 55 & 0 & 0 & -7.2453586 & 112.759951 & 29 \\
\hline 300 & 58 & 0 & 0 & -7.2453586 & 112.759951 & 1 \\
\hline 301 & 40 & 0 & 0 & -7.2453594 & 112.759952 & 1 \\
\hline 303 & 82 & 0 & 0 & -7.2453594 & 112.759952 & 0 \\
\hline
\end{tabular}

\subsection{Clustering Process}

Learning process is conducted by K-Means algorithm. Processed data is the data pattern results from the feature extraction process. Data pattern has five dimensions (time, CPU, upload, download, latitude, longitude). The data is used because it has a high correlation to the user activity. CPU, upload, and download describes the device condition that is being used or not. Whereas location [8] can describe the activities related to office work or activities at 
home. Activities at the office and at home greatly affect how users handle their smartphone.

Figure 2 is diagram of data learning process that obtained from first process. On these process data clustered into several data groups, then average of idle time is calculated for every cluster. The output of this process is centroid and idle time for every cluster.

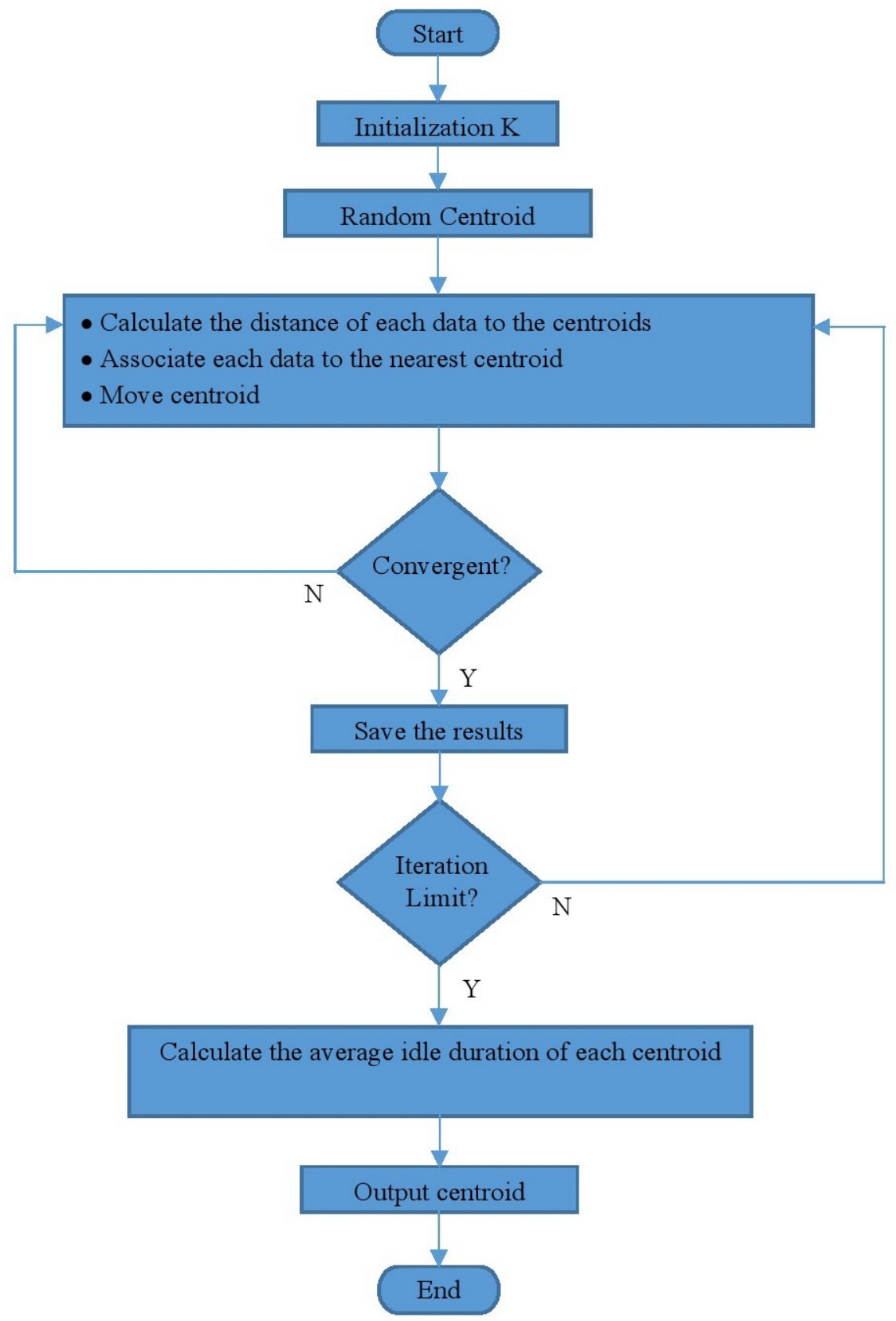

Figure 2. Learning Pattern 


\subsection{Matching Process}

Figure 3 is a flowchart of pattern matching process and giving interval. Matching process conducted by calculating centroid range from previous clustering process. After nearest centroid known, then we get idle time for the data. Then giving interval on internet data traffic based on idle time duration.

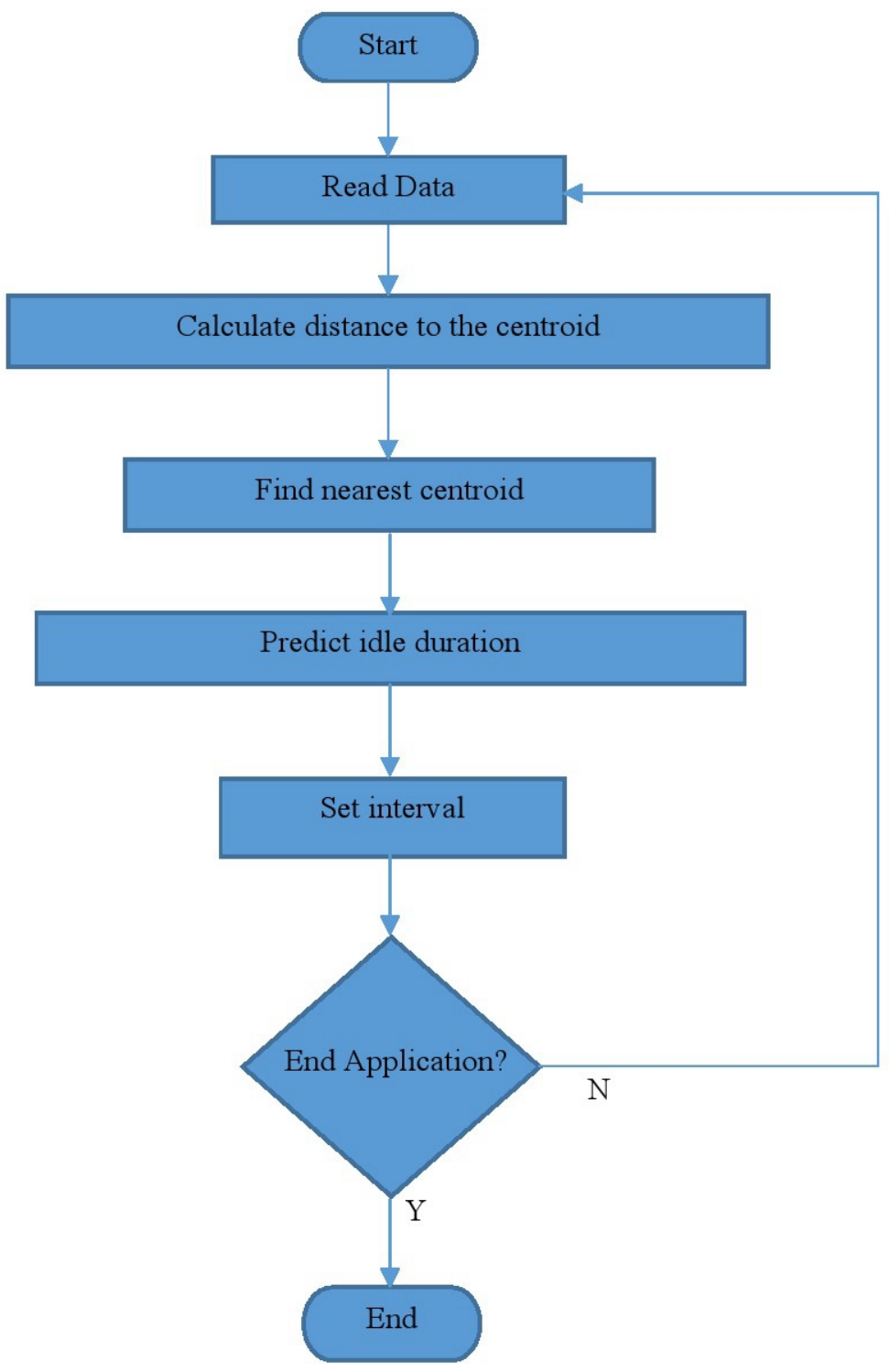

Figure 3. Matching Process

\section{EXPERIMENTS AND ANALYSIS}

Experiment conducted in two scenarios. The first scenarios is aimed to determine the performance of our proposed method in reducing energy consumption. The second scenario aims to determine effectiveness of the method on users with different characteristics. 


\subsection{Battery Life}

This test was conducted to see how much energy consumption can be saved by using our proposed method. This test also carried out a comparison between the proposed method (method A) with other method. The comparison method is an internet connection control method based on idle time without using interval (method B). This method is chosen for comparison because it has similarities to control the internet connection based on idle time. These experiments observed the capacity of battery energy when the smartphone is not using any method. Tests are performed at idle condition with certain idle duration. Battery energy availability was observed when the device start idle state and ends idle state. This is the result of these experiments:

Table 4. Battery Life Time

\begin{tabular}{|c|c|c|c|}
\hline \multirow{2}{*}{$\begin{array}{c}\text { Idle Duration } \\
\text { (minutes) }\end{array}$} & \multicolumn{3}{|c|}{ Energy Consumption (\%) } \\
\cline { 2 - 4 } & Without any methods & Method A & Method B \\
\hline 30 & 2 & 2 & 1 \\
\hline 60 & 4 & 3 & 2 \\
\hline 120 & 8 & 6 & 4 \\
\hline 180 & 12 & 8 & 5 \\
\hline 300 & 20 & 13 & 8 \\
\hline
\end{tabular}

Table 4 is the result of monitoring the availability of the battery energy during idle time. The table shown by using method B there is considerable energy savings, compared to the condition of the device without using any method. When the device using A method (the proposed method) there are also battery energy savings but not as much as method B. This is due to the use of intervals in our method. With the interval on the internet connection control, internet connection alive in a certain time interval in which the internet connection consumes battery energy. While using method $B$, internet connection completely stopped when idle, so there is no energy consumption for the internet connection.

In Table 4 shows, with different idle duration, different battery energy saved in method A or B. The longer the duration of idle occurs greater battery energy saved. It is proved that the amount of energy saved by our proposed method is highly dependent on the length of the idle condition occurs.

\subsection{Users with Different Habits}

This test is aimed to determine effectiveness of proposed approached for users with different habits. Testing was conducted on some users with different habits. Figure 4 is a graph of user activity within one day. 


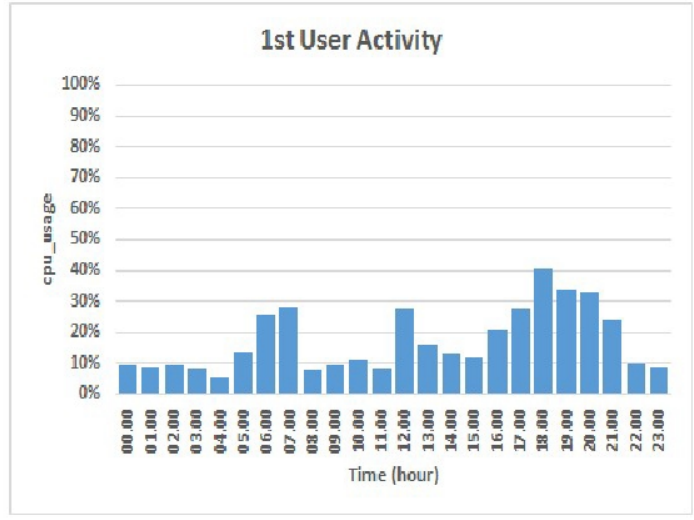

(a)

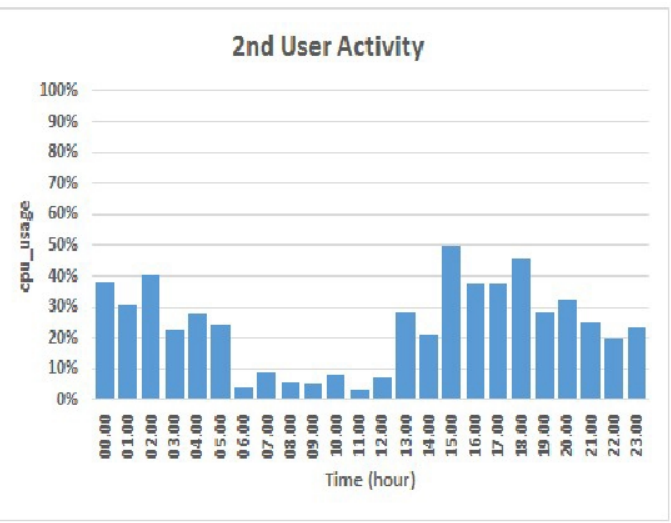

(b)

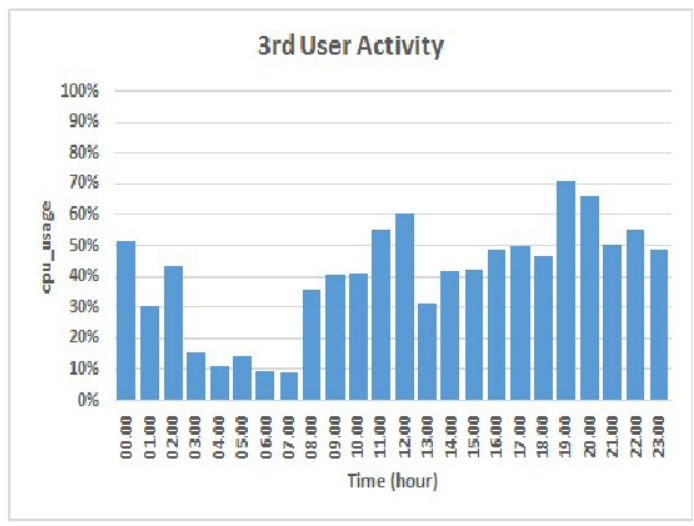

(c)

Figure 4. User Activity Diagram In One Day. (a) User A, (b) User B, (c) User C

Figure 4 shows the differences in the activity of users in one day. Differences in user activity can be observed from the intensity value of the CPU usage. High CPU usage values, indicating at that time the user is actively using a smartphone device. Low CPU usage value, indicating smartphone on idle condition. The higher average CPU usage in one day, indicating that the user is active in the use of smartphones. The following is an explanation for each user.

- User A.

Times with low CPU usage values often occur. Average CPU usage of the activity in one day is low. This means that the users are less active.

- User B.

Times with low CPU usage values often occur. But the average CPU usage of the activity in one day is high. This means that the users are more active Ethan user A.

- User C.

Times with low CPU usage values is rarely happen. Average CPU usage of the activity in one day is very high. This means that the users are very active. 
This test was conducted to see if the system can recognize patterns in accordance with the design of the user habits that have been made. In this test data from each user is processed to obtain a centroid of each user habits. The initial process of pattern recognition is the user's habits data retrieval and feature extraction. The results of this initial process is a pattern of user's habits which have six dimensions. Then this pattern are used for the learning process.

The user data patterns are processed by k-means methods to obtain the centroid of the data. This is the results of the learning process of each user patterns:

Table 5. Users with Different Habits

\begin{tabular}{|c|c|c|c|c|c|c|}
\hline \multicolumn{7}{|c|}{ 1st User Centroid } \\
\hline $\begin{array}{c}\text { Idle } \\
\text { Duration }\end{array}$ & time & CPU & Upload & Download & latitude & Longitude \\
\hline 445 & 5.0019345 & 3.0 & 6.000008 & 9.000044 & -72444176 & 112760077 \\
\hline 427 & 767.5161 & 12.333096 & 27.33291 & 20.66708 & -72450144 & 112760013 \\
\hline 828 & 1271.3285 & 2.847105 & 18.683538 & 21.251959 & -72450712 & 112759987 \\
\hline 54 & 1164.5248 & 1.0201284 & 6.4906 & 16.721407 & -72450880 & 112760050 \\
\hline 280 & 470.00708 & 3.0 & 4.000058 & 8.500236 & -72448144 & 112760038 \\
\hline \multicolumn{7}{|c|}{ 2nd User Centroid } \\
\hline $\begin{array}{c}\text { Idle } \\
\text { Duration }\end{array}$ & time & CPU & Upload & Download & latitude & Longitude \\
\hline 1168 & 1218.572 & 130.84439 & 241.98785 & 94.622 & -72453448 & 112759962 \\
\hline 353 & 1067.8619 & 3.5187466 & 18.431087 & 8.469454 & -72450352 & 112759987 \\
\hline 285 & 765.00226 & 3.0018523 & 2.0033653 & 4.0012774 & -72444176 & 112760077 \\
\hline 1045 & 5.0000124 & 3.0000033 & 32.000008 & 53.000004 & -72351192 & 112766029 \\
\hline 46 & 1005.2059 & 2.4158833 & 5.7492895 & 5.284684 & -72453488 & 112759949 \\
\hline \multicolumn{7}{|c|}{ 3rd User Centroid } \\
\hline $\begin{array}{c}\text { Idle } \\
\text { Duration }\end{array}$ & time & CPU & Upload & Download & latitude & Longitude \\
\hline 212 & 657.4855 & 2.168899 & 25.851843 & 3.840349 & -7245012 & 112759974 \\
\hline 537 & 1175.295 & 89.81117 & 503.48114 & 196.73079 & -72450656 & 112760013 \\
\hline 79 & 507.9427 & 1.3369879 & 19.354736 & 5.4049754 & -72453464 & 112759974 \\
\hline 335 & 105.91693 & 3.0000186 & 26.451122 & 29.298307 & -72448752 & 112760013 \\
\hline 343 & 995.5439 & 3.4866633 & 40.448624 & 29.190819 & -72450824 & 112759987 \\
\hline
\end{tabular}


Table 5 shows the centroid result of the learning process of each user. From each cluster generated, we can see the difference between the users. This difference can be seen from the idle duration in each cluster for each user. The greater the duration of the idle on the resulting clusters indicates that smartphones often enters idle time with a fairly long duration. The smaller the idle duration value in each cluster indicates that users are accustomed to idle in a short duration. Table 5 shows that the 3 rd users are actively using smartphones.

After learning process, then idle duration prediction performed based on centroid results. From the results of these predictions, we can see how effective our method for each user. On this experiment, idle duration prediction result compare to idle duration realization. This is the samples result of the experiments.

Table 6. Idle Duration Prediction

\begin{tabular}{|c|c|c|c|}
\hline No & User & $\begin{array}{c}\text { Actual Idle } \\
\text { Duration }\end{array}$ & $\begin{array}{c}\text { Idle Duration } \\
\text { Predictions }\end{array}$ \\
\hline 1 & \multirow{5}{*}{ 1st User } & 259 & 249 \\
\hline 2 & & 283 & 235 \\
\hline 3 & & 255 & 222 \\
\hline 4 & & 364 & 332 \\
\hline 5 & & 381 & 444 \\
\hline 6 & \multirow{5}{*}{ 2nd User } & 802 & 1045 \\
\hline 7 & & 801 & 1044 \\
\hline 8 & & 290 & 46 \\
\hline 9 & & 371 & 46 \\
\hline 10 & & 1020 & 1167 \\
\hline 11 & \multirow{5}{*}{ 3rd User } & 413 & 344 \\
\hline 12 & & 203 & 335 \\
\hline 13 & & 300 & 349 \\
\hline 14 & & 419 & 525 \\
\hline 15 & & 414 & 345 \\
\hline
\end{tabular}

To see the performance of the prediction of the duration of the idle, then error rate of the idle duration predictions calculated for each user. Table 7 is the result of the error rate calculation of idle duration prediction for each user. The table shows that the lowest error rate is the first. Results prediction idle duration is greatly influenced by the pattern of each user's habits.

Table 7. Idle Duration Prediction Error Rate

\begin{tabular}{|c|c|c|}
\hline No & Users & Error Rate (\%) \\
\hline 1 & 1st User & $19,9 \%$ \\
\hline 2 & 2nd User & $25,3 \%$ \\
\hline 3 & 3rd User & $31 \%$ \\
\hline
\end{tabular}


These experiments prove that the system can perform recognition user habits. The success rate of user habits pattern recognition is influenced by the activity of the user. In addition, this test also proves the functionality of the system to predict idle duration. The accuracy of the prediction results strongly influenced by the learning outcomes of user activity data.

\section{CONCLUSION}

This paper proposes a new approach to saving smartphone battery energy using internet connection control based on idle time. Test results show that our approach is successful in reducing energy consumption on smartphones. The effectiveness of this method is affected by user activity. The more often the device entering idle condition, the more energy is saved. The proposed method has advantages in term of user convenience. When users use the smartphone there is no data transmission delay due to the termination of an internet connection, because the termination of an internet connection only at idle time where the user is not using the device. Besides that periodically data transfer process is still going on when the device is in idle condition.

The average error rate of idle duration prediction results are still high, about 19\% - 30\%. This results is affected by the user habits data and the learning methods are used. In this research we use k-means cluster method to identify user patterns, and predict the duration of idle. For the future work, user behavior recognition can be developed using other methods for more accurate prediction results.

\section{REFERENCES}

[1] Zhang. L, B. Tiwana, R. P. Dick, Z. Qian, Z. M. Mao, Z. Wang, and L. Yang. 2010. Accurate Online Power Estimation and Automatic Battery Behavior Based Power Model Generation for Smartphones. Hardware/Software Codesign and System Synthesis: 105-114.

[2] Zahid, I. M. A. Ali, and R. Nassr. 2011. Android Smartphone: Battery Saving Service. Research and Innovation in Information Systems: 1-4.

[3] Khairy, A., H. H. Ammar, and R. Bahgat. 2013. Smartphone Energizer: Extending Smartphone's Battery Life with Smart Offloading. International Wireless Communications and Mobile Computing Conference: 329-336.

[4] Kim, M. W., D. G. Yun, J. M. Lee, and S. G. Choi. 2012. Battery Life Time Extension Method Using Selective Data Reception on Smartphone. International Conference of Information Networking: 468-471.

[5] Hyeon, Y., M. W. Kim, J. M. Lee, and S. G. Choi. 2012. Battery Life Time Extension Method by Using Signalling Interval Control. International Conference Advanced Communication Technology: 327-330.

[6] Kang, J. M., S. S. Seo, and J. W.-K. Hong. 2011. Usage Pattern Analisys of Smartphones. Network Operations and Management Symposium: 1-8. 
[7] Noulas, A., S. Scellato., C. Mascolo., M. Pontil. 2011. An Empirical Study of Geographic User Activity Patterns in Foursquare. Association for the Advancement of Artificial Intelligence: 570-573.

[8] Farrahi, K., Daniel, G. 2008. Daily Routine Classification from Mobile Phone. Machine Learning for Multimodal Interaction: 173-184. 